OPEN ACCESS

Edited by:

Leonidas H. Duntas,

National University of Athens, Greece

Reviewed by:

Alexander M. Schreiber,

St. Lawrence University, United States

Serena Ippolito,

ASL Napoli 1 Centro, Italy

*Correspondence:

Ming $\mathrm{Li}$

liming@hrbmu.edu.cn

Dian-jun Sun

hrbmusdj@163.com

Specialty section:

This article was submitted to

Thyroid Endocrinology,

a section of the journal

Frontiers in Endocrinology

Received: 09 September 2021

Accepted: 17 November 2021

Published: 09 December 2021

Citation:

Nie G-y, Wang R, Liu P, Li M and Sun D-j (2021) Mild Anemia May Affect Thyroid Function in Pregnant Chinese

Women During the First Trimester.

Front. Endocrinol. 12:772917.

doi: 10.3389/fendo.2021.772917

\section{Mild Anemia May Affect Thyroid Function in Pregnant Chinese Women During the First Trimester}

\author{
Guan-ying Nie ${ }^{1}$, Rui Wang ${ }^{2}$, Peng Liu ${ }^{1}$, Ming $L_{i}{ }^{1 *}$ and Dian-jun Sun ${ }^{1 *}$ \\ ${ }^{1}$ Key Lab of Etiology and Epidemiology, National Health Commission \& Education Bureau of Heilongjiang Province \\ (23618504), Key Laboratory of Trace Elements and Human Health, Center for Endemic Disease Control, Chinese Center for \\ Disease Control and Prevention, Harbin Medical University, Harbin, China, ${ }^{2}$ Examination Department, Central Hospital \\ Affiliated to Shenyang Medical College, Shenyang, China
}

Background: Pregnant women are often susceptible to anemia, which can damage the thyroid gland. However, compared with moderate and severe anemia, less attention has been paid to mild anemia. The purpose of this study was to evaluate the effect of mild anemia on the thyroid function in pregnant women during the first trimester.

Methods: A total of 1,761 women in the first trimester of their pregnancy were enrolled from Shenyang, China, and divided into mild anemia and normal control groups based on their hemoglobin levels. Thyroid-stimulating hormone (TSH), free thyroxine (FT4), and free triiodothyronine (FT3) levels were compared between the two groups.

Results: The TSH levels of pregnant women with mild anemia were higher than those of pregnant women without mild anemia $(p<0.05)$. Normal control women were selected to set new reference intervals for $\mathrm{TSH}, \mathrm{FT} 3$, and FT4 levels during the first trimester, which were $0.11-4.13 \mathrm{mlU} / \mathrm{l}, 3.45-5.47 \mathrm{pmol} / \mathrm{l}$, and $7.96-16.54 \mathrm{pmol} / \mathrm{l}$, respectively. The upper limit of TSH $4.13 \mathrm{mU} / \mathrm{I}$ is close to the upper limit $4.0 \mathrm{mU} / \mathrm{l}$ recommended in the 2017 American Thyroid Association (ATA) guidelines, indicating that exclusion of mild anemia may reduce the difference in reference values from different regions. Mild anemia was related to 4.40 times odds of abnormally TSH levels (95\% Cl: $2.84,6.76)$ and 5.87 increased odds of abnormal FT3 (95\% Cl: 3.89, 8.85). The proportion of hypothyroidism and subclinical hypothyroidism in patients with mild anemia was higher than that in those without anemia (0.6\% vs. $0, p=0.009 ; 12.1 \%$ vs. $1.9 \%, p<0.001)$. Mild anemia was related to 7.61 times increased odds of subclinical hypothyroidism (95\% Cl: 4.53, 12.90).

Conclusions: Mild anemia may affect thyroid function during the first trimester, which highlights the importance of excluding mild anemia confounding when establishing a locally derived specific reference interval for early pregnancy.

Keywords: the first trimester, mild anemia, FT4, TSH, reference values, subclinical hypothyroidism 


\section{INTRODUCTION}

Thyroid dysfunction during pregnancy increases the risk of miscarriage, premature birth, fetal malformation, and even fetal death $(1,2)$. Therefore, to maintain maternal and child health, thyroid function should be actively advocated for all pregnant women.

Anemia is defined as a hemoglobin level of $120 \mathrm{~g} / \mathrm{l}$ or less in women, and $110 \mathrm{~g} / \mathrm{l}$ or less in pregnant women (3-5). The World Health Organization (WHO) has reported that $23 \%$ of pregnant women in industrialized countries and $52 \%$ in non-industrialized countries suffer from anemia (6). Iron deficiency (ID) is the main cause of anemia (7). Other causes include lack of vitamin A, B12, or folic acid; infectious diseases such as malaria and AIDS; and other hereditary anemias such as sickle cell disease (8). Anemia and thyroid function studies carried out in animal models have verified that low iron intake significantly decreases hemoglobin (Hb) levels, thyroid peroxidase (TPO) activity $(9,10)$, and serum concentrations of T3 and T4 (11), while increasing TSH levels (12). In 2007, Zimmermann et al. first reported that ID was associated with higher TSH and lower T4 levels in Swiss pregnant women (13). In recent years, studies in China have shown that ID can also be associated with abnormal thyroid function of pregnant women (14-17). Additionally, hypothyroidism occurs in patients with chronic hemolytic anemia, and its incidence is positively correlated with age and the severity of anemia (18).

Nevertheless, insufficient attention has been directed toward the relationship between mild anemia and thyroid function in pregnant women. Mild, moderate, and severe anemia in women was defined as a hemoglobin level of $110-119,80-109$, and $<80$ $\mathrm{g} / \mathrm{l}$, while that in pregnant women was defined as 100-109, 7099 , and $<70 \mathrm{~g} / \mathrm{l}(3-5)$. A study has revealed that pregnant women with mild, moderate, or severe anemia are related to infant anemia (19). Compared to those of moderate and severe anemia, the symptoms of mild anemia are non-specific and are only clinically detectable, which might explain why little attention is paid to mild anemia. A study has reported the prevalence of anemia among Chinese women before pregnancy to be $21.64 \%$ (mild: $14.10 \%$, moderate: $7.17 \%$, and severe: $0.37 \%$ ) in 2017 (20). According to the results monitored from 2010 to 2012 , the anemia rate in pregnant women in China was $17.2 \%$, while mild anemia accounted for approximately $61.0 \%$ (21). Mild anemia accounted for the highest proportion of overall anemia. Therefore, this study aimed to explore the effect of mild anemia in early pregnancy on the thyroid function of pregnant women in Shenyang, a city in northeast of China.

\section{MATERIALS AND METHODS}

\section{Study Population}

The study was approved by the Ethics Committee of Harbin Medical University. We extracted the first routine evaluation results of pregnant women in the obstetric outpatient department of the Affiliated Central Hospital of Shenyang Medical College from September 2011 to December 2018. Prior to enrollment into the study, the pregnant women underwent routine blood and thyroid function examination $(n=2,902)$. Women with a pregnancy of 10-12 weeks of gestation were included in the study. Those women with a pregnancy of less than 10 weeks or more than 12 weeks of gestation were not eligible to participate $(n=712)$. Pregnant women with moderate anemia, severe anemia, self-reported blood diseases, infections, fever, drug treatment, or supplement treatment $(n=129)$ were also excluded. Pregnant women with self-reported thyroid disease $(\mathrm{n}=154)$ or positive thyroid autoantibodies (TPOAb, TgAb, or TMAb) $(\mathrm{n}=$ 146) were also considered to be ineligible. Based on the abovementioned criteria, a total of 1,761 pregnant women were selected, including 314 pregnant women with mild anemia and 1,447 pregnant women without anemia as the control group (Figure 1).

\section{Method}

Venous blood samples were collected in the morning after overnight fasting for more than $8 \mathrm{~h}$. Blood samples were centrifuged to obtain serum, which was sent to the clinical laboratory for testing. The levels TSH, FT4, and FT3 were determined in all subjects on the same day of sampling, using a commercial electrochemiluminescence immunoassay kit (Beckman UniCel DxI800 automatic chemiluminescence analyzer, USA). Hemoglobin ( $\mathrm{Hb}$ ) levels were measured using an automated hematology analyzer XE-2100 (Sysmex Diagnostics, Japan). The women were considered to have mild anemic if their $\mathrm{Hb}$ was determined to be in the range 100-109 g/l.

\section{Statistical Analysis}

Statistical analysis was performed using the Statistical Product and Service Solutions (SPSS) software (version 17.0, Chicago, IL). The Kolmogorov-Smirnov test was performed to confirm normality. Normally distributed FT4 were represented as mean

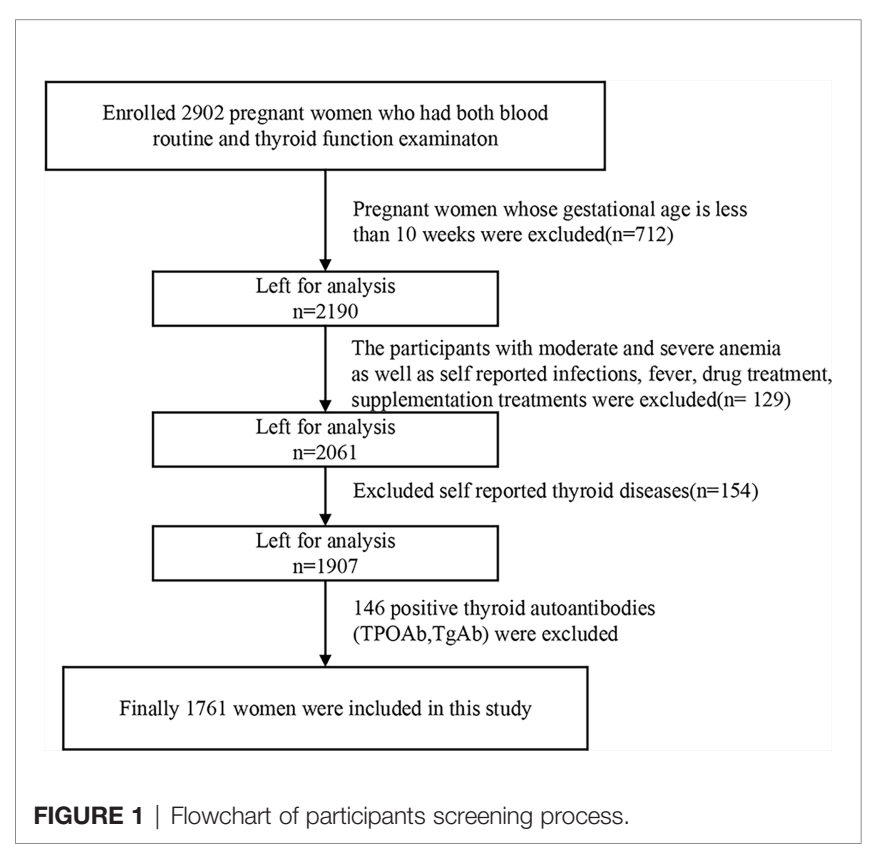


\pm standard deviation (SD), while FT3 and TSH did not follow a Gaussian distribution which were represented by a median (minimum-maximum). For normally distributed data, a t-test was applied to compare groups. For non-normal data, a KruskalWallis test and subsequent Mann-Whitney test were used to compare groups. When comparing the rates between groups, we use the chi-square test. We used univariable and multivariate logistic regression to examine the significant influence factors for thyroid function. A two-tailed p-value $<0.05$ was considered statistically significant. Statistical power analyses were done using G*Power 3.1 (22). According to NCCLS C28-A2, the 2.5th and 97.5th percentiles were considered as the lower and upper limits, respectively, of the reference intervals (23). The 95\% confidence interval (CI) of the two limits was obtained through bootstrap by $\mathrm{R} 4.0 .2$.

\section{RESULTS}

\section{Subject Characterization}

In total, 1,761 individuals with a mean age of $29.37 \pm 3.84$ years participated in this study. The average gestation age was $11 \pm 1$ week. The median TSH level was $1.6 \mathrm{mIU} / \mathrm{l}$ (range 0.01, 9.67 $\mathrm{mIU} / \mathrm{l}$ ), the median FT4 level was $12.57 \mathrm{pmol} / \mathrm{l}$ (range 1.15, 23.06 $\mathrm{pmol} / \mathrm{l}$ ), and the median FT3 level was $4.26 \mathrm{pmol} / \mathrm{l}$ (range 2.67, $13.15 \mathrm{pmol} / \mathrm{l})$. The prevalence of mild anemia among all women was $17.8 \%(314 / 1761)$.

\section{Different Distribution of Thyroid Hormones During the First Trimester Between Women With Mild Anemic and Control Pregnant Women}

We compared the thyroid function values between pregnant women with mild anemia and the control group and found that there was no difference in the levels of FT3 and FT4 between the two groups. The TSH levels in pregnant women with mild anemia were higher than those in pregnant women without anemia $(\mathrm{p}<0.001)$, as shown in Table 1 .

\section{Reference Range for Thyroid Hormones During the First Trimester in Pregnant Women}

We choose pregnant women in the control group to set a new reference interval. As shown in Table 2, the TSH ranges from 0.11 to $4.13 \mathrm{mIU} / \mathrm{l}, \mathrm{FT} 3$ from 3.45 to $5.47 \mathrm{pmol} / \mathrm{l}$, and FT4 from 7.96 to
$16.54 \mathrm{pmol} / \mathrm{l}$. The standard reference ranges provided by manufacturers are as follows: TSH ranges from 0.68 to 5.59 $\mathrm{mIU} / \mathrm{l}$, FT3 from 3.28 to $6.47 \mathrm{pmol} / \mathrm{l}$, and FT4 from 7.64 to $16.03 \mathrm{pmol} / \mathrm{l}$. We selected pregnant women from both the control group and the group comprising women with mild anemia to calculate a reference interval, wherein the range of TSH was considered to be from 0.13 to $4.65 \mathrm{mIU} / \mathrm{l}$, FT3 from 3.43 to 5.91 $\mathrm{pmol} / \mathrm{l}$, and FT4 from 7.98 to $16.54 \mathrm{pmol} / \mathrm{l}$.

On comparing our new reference with the standard references, serum TSH levels were decreased in the first trimester, with the upper limit declining by $26.2 \%$ and the lower limit declining by $83.4 \%$. The upper limit of serum FT3 declined by $15.5 \%$, and the lower limit increased by $5.2 \%$. Serum FT4 showed an increase too, with the upper and lower limits raised by $3.2 \%$ and $4.2 \%$, respectively.

Comparison between the new reference with the references including mild anemia demonstrated the following changes. Serum TSH levels decreased in the first trimester, with the upper limit declining by $11.1 \%$ and the lower limit declining by $15.4 \%$, while the serum FT3 upper limit declined by $7.5 \%$.

\section{TSH and FT3 but Not FT4 May Be Affected by Mild Anemia in Early Pregnancy}

Based on the reference range of healthy control pregnant women, we compared the indicators of thyroid function between pregnant women with mild anemia and the control group. As shown in Table 3, the rate of TSH values above the upper limit in pregnant women with mild anemia was notably higher than that in the control $(12.7 \%$ vs. $1.9 \%, p<0.0001)$. The percent of FT3 values under the lower limit or above the upper limit in pregnant women with mild anemia was greater than that in the control (3.8\% vs. $1.9 \%, 15.0 \%$ vs. $1.9 \%, p<0.0001)$. In addition, there was no significant difference between the FT4 values.

As shown in Table 5, mild anemia [OR 4.26 (95\% CI: 2.82, $6.43) ; \mathrm{p}<0.001)$ ] was independently associated with a higher risk for abnormal TSH levels. After adjustment for confounding variables in the final step forward logistic regression, mild anemia (OR 4.40 (95\% CI: 2.84, 6.76); p < 0.001) was independently associated with a higher risk for abnormal TSH levels. Mild anemia (OR 5.86 (95\% CI: 3.96, 8.67); $p<0.001$ ) was independently associated with a higher risk for FT3 abnormality risk. In addition, age $>35$ years (OR 2.45 (95\% CI: 1.30, 4.33); $p<$ 0.001 ) was associated with a higher FT3 abnormality risk. After adjustment for confounding variables in the final step forward logistic regression, mild anemia (OR 5.87 (95\% CI: 3.89, 8.85);

TABLE 1 | Comparison of thyroid functions between pregnant women with mild anemia and control group.

\begin{tabular}{|c|c|c|c|}
\hline \multirow[t]{2}{*}{ Thyroid function } & \multicolumn{2}{|c|}{ Pregnant women } & \multirow[t]{2}{*}{ p value } \\
\hline & Control $(n=1447)$ & Mild anemia $(n=314)$ & \\
\hline FT3 (pmol/L), median [min, max] & $4.24[2.67,5.94]$ & $4.35[3.11,13.15]$ & $0.05^{a}$ \\
\hline FT4 (pmol/L), mean \pm SD & $12.43 \pm 2.29$ & $12.49 \pm 2.34$ & $0.655^{b}$ \\
\hline TSH (mIU/L), median [min, max] & $1.58[0.01,6.47]$ & $1.72[0.01,9.67]$ & $<0.001^{a *}$ \\
\hline
\end{tabular}

a Mann-Whitney U-test was used to compare the groups.

${ }^{b} T$-test was used to compare the groups.

*Statistical significance was assumed when the $p$-value was $<0.05$. 
TABLE 2 | Reference ranges of thyroid function.

\begin{tabular}{|c|c|c|c|c|c|}
\hline Thyroid function & $\mathbf{n}$ & P2.5 & $95 \% \mathrm{Cl}$ (P 2.5) & P 97.5 & $95 \% \mathrm{Cl}$ (P 97.5) \\
\hline \multicolumn{6}{|l|}{ FT3 (pmol/L) } \\
\hline Standard & & 3.28 & & 6.47 & \\
\hline Pregnant women & 1761 & 3.43 & $3.39-3.46$ & 5.91 & $5.81-6.36$ \\
\hline New & 1447 & 3.45 & $3.41-3.49$ & 5.47 & $5.42-5.56$ \\
\hline \multicolumn{6}{|l|}{ FT4 (pmol/L) } \\
\hline Standard & & 7.64 & & 16.03 & \\
\hline Pregnant women & 1761 & 7.98 & $7.85-8.16$ & 16.54 & $16.27-16.90$ \\
\hline New & 1447 & 7.96 & 7.79-8.09 & 16.54 & $16.28-16.86$ \\
\hline \multicolumn{6}{|l|}{ TSH (mlU/L) } \\
\hline Standard & & 0.68 & & 5.59 & \\
\hline Pregnant women & 1761 & 0.13 & $0.06-0.44$ & 4.65 & $4.56-5.03$ \\
\hline New & 1447 & 0.11 & $0.07-0.18$ & 4.13 & $3.98-4.26$ \\
\hline
\end{tabular}

Standard, the reference ranges provided by manufacturers.

Pregnant women, pregnant women from both the control group as well as the group comprising women with mild anemia were selected to calculate a reference interval.

New, only chose control pregnant women to set the new reference interval.

TABLE 3 | Comparison of thyroid functions abnormal between pregnant women with mild anemia and control group.

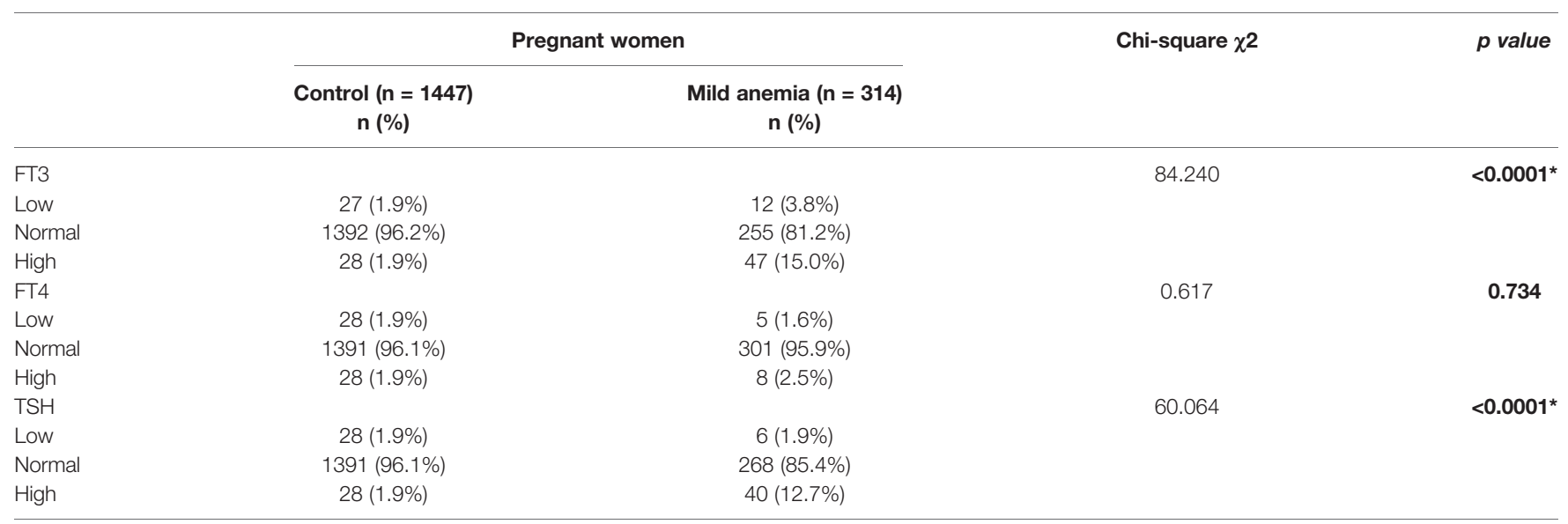

*Statistical significance was assumed when the $p$-value was $<0.05$.

$p<0.001)$ was independently associated with a higher FT3 abnormality risk. Age $>35$ years was no longer associated with a higher risk for FT3 abnormal.

\section{Mild Anemia May Be Related to Subclinical Hypothyroidism in Early Pregnancy}

As shown in Table 4, the prevalence of subclinical hypothyroidism was markedly higher in pregnant women with mild anemia than in the control group $(12.1 \%$ vs. $1.9 \%, p<0.001)$. Moreover, there were two $(0.64 \%)$ overt hypothyroidism cases in pregnant women with mild anemia, but none was found in the control group. These differences were significant $(p=0.009)$. In contrast, there was no significant difference in the prevalence of subclinical hyperthyroidism and hyperthyroidism between the pregnant women with mild anemia and the controls. As shown in Table 5, mild anemia (OR 6.98 (95\% CI: 4.23, 11.65); p < 0.001)) was independently associated with a higher risk for subclinical hypothyroidism. After adjustment for confounding variables in the final step forward logistic regression, mild anemia (OR 7.61 (95\% CI: 4.53, 12.90); p < 0.001) was independently associated with a higher risk for subclinical hypothyroidism.

\section{DISCUSSION}

Anemia is a common disease that may occur during pregnancy. A meta-analysis study from China demonstrated that the prevalence of anemia in pregnant women during the first trimester was found to be $10.1 \%$ (95\% CI $6.2 \%-14 \%$ ) during the period from 2012 to 2016 (24). The monitoring results from 2011 to 2012 showed that the anemia rate of pregnant women in small and medium-sized Chinese cities is $18.0 \%$ (25). A study has also proven that the risk of anemia in pregnant women in the north is higher than that in the south region of China (21). Mild anemia accounted for the highest proportion of overall anemia. Our study comprised data from women in early pregnancy from February 2012 to December 2018 in Tiexi District of Shenyang and found a mild anemia rate of $17.8 \%$ in pregnant women during their first trimester. Our study found that the high incidence of mild anemia was closely related to higher maternal age (older than 35 years) [OR 10.88 (95\% CI: 7.02, 17.12)] (data not shown). This finding is similar to that reported by Lin et al. Their study surveyed 43,403 pregnant women in Beijing, Chengdu, and Guangzhou and found that maternal anemia was significantly related to maternal age 35 years and older $(\mathrm{AOR}=$ 
TABLE 4 | Prevalence of thyroid diseases in pregnant women with mild anemia compared to the control group.

\begin{tabular}{|c|c|c|c|c|}
\hline & \multicolumn{2}{|c|}{ Pregnant women } & \multirow[t]{2}{*}{ Chi-square $\chi^{2}$} & \multirow[t]{2}{*}{$p$ value } \\
\hline & $\begin{array}{c}\text { Control }(n=1447) \\
n(\%)\end{array}$ & $\begin{array}{c}\text { Mild anemia }(n=314) \\
n(\%)\end{array}$ & & \\
\hline Overt hypothyroidism, n (\%) & & & 6.907 & $0.009^{*}$ \\
\hline Yes & $0(0.0)$ & $2(0.6)$ & & \\
\hline No & 1447 (100.0) & $312(99.4)$ & & \\
\hline Subclinical hypothyroidism, n (\%) & & & 54.903 & $<0.0001^{*}$ \\
\hline Yes & $28(1.9)$ & $38(12.1)$ & & \\
\hline No & $1419(98.1)$ & $276(87.9)$ & & \\
\hline Overt hyperthyroidism, n (\%) & & & 0.416 & 0.519 \\
\hline Yes & $2(0.1)$ & $1(0.3)$ & & \\
\hline No & 1445 (99.9) & $313(99.7)$ & & \\
\hline Subclinical hyperthyroidism, n (\%) & & & 0.064 & 0.800 \\
\hline Yes & $26(1.8)$ & $5(1.6)$ & & \\
\hline No & $1421(98.2)$ & $309(98.4)$ & & \\
\hline
\end{tabular}

*Statistical significance was assumed when the $p$-value was $<0.05$.

TABLE 5 | Results from the univariable and multivariable logistic regression analysis.

\begin{tabular}{|c|c|c|c|c|}
\hline \multirow{2}{*}{$\begin{array}{l}\text { Dependent/independent } \\
\text { variables }\end{array}$} & \multicolumn{2}{|c|}{ Univariable analysis } & \multicolumn{2}{|c|}{ Multivariable analysis } \\
\hline & Rough OR (95\% Cl) & $p$ value & Adjust OR ${ }^{a}(95 \% \mathrm{Cl})$ & $p$ value \\
\hline \multicolumn{5}{|c|}{ Outcome: abnormal FT3 levels (logistic regression) } \\
\hline Mild anemia & $5.86(3.96,8.67)$ & $<0.0001^{*}$ & $5.87(3.89,8.85)$ & $<0.0001^{*}$ \\
\hline Age group (>35) & $2.45(1.30,4.33)$ & $<0.0001^{*}$ & & \\
\hline \multicolumn{5}{|c|}{ Outcome: abnormal FT4 levels (logistic regression) } \\
\hline Age group (>35) & $0.72(0.17,1.99)$ & 0.589 & & \\
\hline Mild anemia & $1.07(0.55,1.92)$ & 0.823 & & \\
\hline \multicolumn{5}{|c|}{ Outcome: abnormal TSH levels (logistic regression) } \\
\hline Mild anemia & $4.26(2.82,6.43)$ & $<0.0001^{*}$ & $4.40(2.84,6.76)$ & $<0.0001^{*}$ \\
\hline Age group (>35) & $1.83(0.87,3.47)$ & 0.08 & & \\
\hline \multicolumn{5}{|c|}{ Outcome: subclinical hypothyroidism (logistic regression) } \\
\hline Mild anemia & $6.98(4.23,11.65)$ & $<0.0001^{*}$ & $7.61(4.53,12.90)$ & $<0.0001^{*}$ \\
\hline Age group (>35) & $1.65(0.62,3.62)$ & 0.25 & & \\
\hline
\end{tabular}

*Statistical significance was assumed when the $p$-value was $<0.05$.

${ }^{a}$ Adjust for age.

1.386) (26). Pregnancies at an advanced reproductive age are common now. Therefore, it is essential to give additional importance to the influence of mild anemia as well as anemia on health of pregnant women and fetus.

Fifty percent of overall anemia cases in populations is caused by ID (8). ID is frequent during the first trimester of pregnancy and is often related to a higher prevalence of thyroid autoimmunity, increased TSH, and lower FT3 levels (27). A study in Wuxi also confirmed that low iron stores showed a trend toward higher TSH, lower FT3, and lower FT4 levels during the first trimester of pregnancy (15). A study in Suzhou revealed that pregnant women with mild ID and ID anemia have higher TSH and lower FT4 status (16). These studies on TSH and FT3 are consistent with our study. In our study, we found that TSH levels were higher in pregnant women with mild anemia than in those without anemia $(\mathrm{p}<0.001)$. According to our new intervals, we observed that mild anemia was independently associated with a higher risk for abnormal TSH and FT3 levels. In this study, we also found that the prevalence of subclinical hypothyroidism and overt hypothyroidism was markedly higher in women with mild anemia than in those without.
However, no differences were observed in FT4 values between pregnant women with mild anemia and those without it. The first reason may be that we excluded pregnant women with selfreported thyroid disease or positive thyroid autoantibodies during sample screening. These patients are the main contributors to the abnormal FT4 levels, which was standard for diagnosis of the thyroid disease. The second reason may be that previous studies focus on ID which includes not only mild anemia but also moderate and severe anemia. However, we just focus on mild anemia. Thirdly, lower FT4 levels may also be related to other factors such as iodine nutrition, age, and gestational weeks, which need to be further elucidated. Ipek et al. found no difference in FT4 levels between ID anemia children and normal children (28). Tienboon and Unachak found that there was no difference in T4, T3, fT4, fT3, thyroxine-binding globulin (TBG), and TSH levels before and after iron treatment in ID anemia children (29). Ravanbod et al. reported the absence of significant differences in $\mathrm{Hb}$ and TSH levels before and after 90 days of iron treatment in non-pregnant patients with ID anemia and subclinical hypothyroidism $(\mathrm{SCH})$, which suggested that iron alone does not change the TSH level in 
non-pregnant patients with ID anemia and SCH (30). Infusion of concentrated red blood cells can increase $\mathrm{Hb}$, thyroxine (T3), and free-T3 (FT3) levels in patients with thalassemia who possessed normal thyroid function before puberty, but it has no effect on patients with delayed puberty (31). Therefore, mild anemia might not be the only determining factor affecting thyroid function.

In our study, the temporality of the association between anemia and abnormal thyroid function could not be assessed because exposure and outcome were measured simultaneously. Nevertheless, anemia and thyroid function animal studies have demonstrated that low-iron food significantly decreased the $\mathrm{Hb}$ levels and TPO activity $(9,10)$, as well as serum concentrations of T3 and T4 (11), while a rise in TSH levels was observed (12). ID anemia resulted in maternal hypothyroxinemia from midgestation to the end of the pregnancy in pregnant rats (32). Interestingly, in Nepalese children, ID was also found to decrease the activity of TPO, an iron-containing enzyme involved in the synthesis of thyroid hormones (33). Iron therapy studies have implied that in ID adolescent girls, improvement in iron status was accompanied by an improvement in some indices of thyroid hormones (34). After treatment with iron, FT4 levels significantly increased in patients with ID anemia (35). Beard et al. reported that in women with ID anemia, iron supplementation corrected the anemia significantly $(p=0.03)$ improved the rectal temperature, and partially normalized the plasma thyroid hormone concentrations (36). Studies have emphasized that adding iron to thyroxine therapy improves both conditions compared to thyroxine therapy alone $(30,37)$. In addition, patients with chronic hemolytic anemia requiring repeated blood transfusion have a high prevalence of the hypothalamic-pituitary thyroid axis. Proper blood transfusion appears to prevent deterioration of thyroid function and, in many cases, can reverse thyroid pathology (18). Therefore, the significant auxiliary effect of mild anemia on thyroid function should not be ignored.

For the evaluation of thyroid function in pregnant women, there have been several studies and guidelines indicating that non-pregnant reference intervals for thyroid hormones are not applicable for pregnancy. There are many challenges in reference value formulation for thyroid function in pregnant women. Maternal human chorionic gonadotrophin (hCG) directly stimulates the TSH receptor, increasing thyroid hormone production by nearly $50 \%$, resulting in a subsequent reduction in serum TSH concentration (38). The levels of thyroid hormones vary according to the gestational age (39). Moreover, the pregnancy reference intervals could be affected by race, kits, and test methods (40-42). It has been reported that the formulation of gestational reference ranges is always given to be inconsistent. The 2011 ATA guidelines suggested a specific upper limit cutoff $(2.5 \mathrm{mU} / \mathrm{l})$ for serum TSH levels in the first trimester of pregnancy (43). Nonetheless, the TSH upper limits given by studies worldwide are higher than $2.5 \mathrm{mU} / \mathrm{l}$. Then, the 2017 ATA guidelines suggested an upper reference limit of 4.0 $\mathrm{mU} / \mathrm{l}$ (1). Nevertheless, a series of studies have demonstrated that compared to the European and American population, or the reference value suggested by ATA, the Chinese pregnant population has a higher upper TSH limit (43-45).

As described above, our study implied that pregnant women with mild anemia had abnormally high TSH levels. According to ATA recommendation, making thyroid function reference ranges should only include pregnant women with no known thyroid disease, optimal iodine intake, and negative thyroid peroxidase antibody (TPOAb) status (1). Conversely, they did not consider excluding pregnant women with mild anemia by clinically $\mathrm{Hb}$ detection. In our study, we excluded mild anemia and established a new first trimester-specific reference interval for pregnant women. Interestingly, the upper limit of $4.13 \mathrm{mU} / \mathrm{l}$ obtained was almost equal to the upper limit of $4.0 \mathrm{mU} / \mathrm{l}$ given by the 2017 ATA guideline (1). Therefore, inconsistent reference ranges worldwide may not only be due to race and iodine nutrition but also be due to the diverse degrees of mild anemia rate among different countries. Pregnant women with mild anemia are easily overlooked due to their symptoms which are non-specific and obscure. In addition, pregnant women with mild anemia during the first trimester may be healthy before pregnancy, and some symptoms of mild anemia are similar to general pregnancy symptoms. Therefore, mild anemia cannot be ruled out without routine blood examinations.

After excluding mild anemia, the upper and lower limits of serum TSH decreased. Therefore, if the reference interval is calculated with anemia confounding, the diagnosis of high TSH levels related to hypothyroidism in pregnant women will be missed, and the diagnosis of lower TSH levels related to hyperthyroidism in pregnant women will be misdiagnosed. After excluding mild anemia, the serum FT3 upper reference limit declined by $7.5 \%$. Thus, if reference values were calculated with mild anemia included, the diagnosis of high FT3 levels related to thyroid function in pregnant women would be missed.

In addition, according to the 2017 ATA guidelines, the upper $\mathrm{TSH}$ limit of $4.0 \mathrm{mU} / 1$ represents a reduction of approximately $0.5 \mathrm{mU} / \mathrm{l}$ from the non-pregnant upper limit (1). The new reference value of $4.13 \mathrm{mU} / \mathrm{l}$ from our study represents a relatively decent rate of reduction (26\%) from the nonpregnant TSH upper reference limit $(5.59 \mathrm{mIU} / \mathrm{l})$ rather than a reduction of $0.5 \mathrm{mU} / \mathrm{l}$. A systematic review by Gao et al. also emphasized that pregnant women had a $22 \%$ reduction in the serum TSH upper limit from the non-pregnant value (42). This can be used as a suboptimal method to determine the threshold value for pregnant women in the first trimester.

It should be mentioned that the present study has several limitations, which need to be improved in further studies. First, certain parameters such as serum ferritin (SF), soluble transferrin receptor (sTfR), and total body iron (TBI) were not measured; the mild anemia status assessment would be more accurate with the measurement of these parameters. Secondly, this hospitalbased study prevented us from obtaining detailed information on lifestyle factors and dietary habits, which may have a critical effect on the causal relationship between maternal thyroid function and mild anemia. Thirdly, pregnant women analyzed in the present study were not randomly selected, and the sample sizes of control and mild anemia groups were not equal, possibly 
introducing a selection bias. Although there is sufficient statistical power to investigate the association between abnormal thyroid function risk and anemia (see in Supplementary Table 1), larger prospective and different phases of pregnancy trials based on multicenters or community to replicate these findings are needed in the future. Fourth, the cross-sectional design of measuring exposure and results at the same time is the main limitation of this study; hence, it is impossible to assess the timeliness of the association between anemia and abnormal thyroid function. Therefore, in order to better understand the impact of mild anemia on thyroid function, the molecular mechanism should be studied based on animal models with different degrees of anemia during pregnancy and cell experiments in the future.

In conclusion, this study suggested a possible association between mild anemia and abnormal thyroid function in pregnant women during the first trimester; therefore, physicians should be aware of mild anemia during the first trimester to avoid adverse pregnancy outcomes. Moreover, the interval made by pregnant women without mild anemia is closer to the 2017 ATA reference, which indicated that the difference in TSH value in pregnant women globally might be partly due to the different incidence rates of mild anemia in pregnant women around the world. Therefore, formulation of thyroid hormones reference for pregnant women should exclude those with mild anemia, which need further study.

\section{DATA AVAILABILITY STATEMENT}

The original contributions presented in the study are included in the article/Supplementary Material. Further inquiries can be directed to the corresponding authors.

\section{REFERENCES}

1. Alexander EK, Pearce EN, Brent GA, Brown RS, Chen H, Dosiou C, et al. 2017 Guidelines of the American Thyroid Association for the Diagnosis and Management of Thyroid Disease During Pregnancy and the Postpartum. Thyroid (2017) 27:315-89. doi: 10.1089/thy.2016.0457

2. Nazarpour S, Ramezani TF, Simbar M, Azizi F. Thyroid Dysfunction and Pregnancy Outcomes. Iranian J Reprod Med (2015) 13:387-96.

3. Organization WH. Haemoglobin Concentrations for the Diagnosis of Anaemia and Assessment of Severity. (2011). Available at: https://www. who.int/vmnis/indicators/haemoglobin.pdf.

4. Cappellini M, Motta I. Anemia in Clinical Practice-Definition and Classification: Does Hemoglobin Change With Aging? Semin Hematol (2015) 52:261-9. doi: 10.1053/j.seminhematol.2015.07.006

5. Method for Anemia Screen WS/T 441-2013. In: N.H.C.o.t.P.s.R.o. China.

6. Lopez A, Cacoub P, Macdougall IC, Peyrin-Biroulet L. Iron Deficiency Anaemia. Lancet (2016) 387:907-16. doi: 10.1016/S0140-6736(15)60865-0

7. WHO/UNICEF/UNU. Iron Ddeficiency Anemia: Assessment, Prevention and Control-a Guide for Program Managers. Geneva Switzerland: Who (2001). p. 1-132.

8. Yip R. Iron Deficiency: Contemporary Scientific Issues and International Programmatic Approaches. J Nutr (1994) 124:1479S-90S. doi: 10.1093/jn/ 124.suppl_8.1479S

9. Hess SY, Zimmermann MB, Arnold M, Langhans W, Hurrell RF. Iron Deficiency Anemia Reduces Thyroid Peroxidase Activity in Rats. J Nutr (2002) 132:1951-5. doi: 10.1093/jn/132.7.1951

\section{ETHICS STATEMENT}

The studies involving human participants were reviewed and approved by the Ethics Committee of Harbin Medical University. The patients/participants provided their written informed consent to participate in this study.

\section{AUTHOR CONTRIBUTIONS}

$\mathrm{ML}$ and $\mathrm{D}$-jS were involved in conception and design of the research. ML drafted the manuscript. G-yN and RW performed the experiments and analyzed the data. G-yN and ML prepared the figures and tables. ML interpreted the results of the experiments. G-yN, RW, PL, ML, and D-jS edited and revised the manuscript and approved the final version of the manuscript.

\section{FUNDING}

This study was supported by the Heilongiiang Natural Science Foundation (Project TD2019H001) and Harbin Medical University Funding (Project HMUMIF21015).

\section{SUPPLEMENTARY MATERIAL}

The Supplementary Material for this article can be found online at: https://www.frontiersin.org/articles/10.3389/fendo.2021.7 72917/full\#supplementary-material

Supplementary Table 1 I (total sample size $=1761$, two-side alpha $=0.05$ ). ${ }^{\text {a }}$ Allocation ratio equal to $14.4474,16.2647$ and 25.68 for FT3, TSH and Subclinical hypothyroidism, respectively.

10. Dillman E, Gale C, Green W, Johnson DG, Mackler B, Finch C. Hypothermia in Iron Deficiency Due to Altered Triiodothyronine Metabolism. Am J Physiol (1980) 239:377-81. doi: 10.1152/ajpregu.1980.239.5.R377

11. Smith SM, Johnson PE, Lukaski HC. In Vitro Hepatic Thyroid Hormone Deiodination in Iron-Deficient Rats: Effect of Dietary Fat. Life Sci (1993) 53:603-9. doi: 10.1016/0024-3205(93)90268-8

12. Mathur N, Joshi SC, Mathur S. Effect of Dietary Iron Deficiency Anaemia on TSH and Peripartum Thyroid Function. BioScientifica (2006) 12:123. doi: 10.5005/jp/books/10766_74

13. Zimmermann MB, Hans B, Hurrell RF. Iron Deficiency Predicts Poor Maternal Thyroid Status During Pregnancy. J Clin Endocrinol Metab (2007) 3436-40. doi: 10.1210/jc.2007-1082

14. Teng X, Shan Z, Li C, Yu X, Mao J, Wang W, et al. Iron Deficiency May Predict Greater Risk for Hypothyroxinemia: A Retrospective Cohort Study of Pregnant Women in China. Thyroid (2018) 28:968-75. doi: 10.1089/ thy.2017.0491

15. Fu J, Yang A, Zhao J, Zhu Y, Chen D. The Relationship Between Iron Level and Thyroid Function During the First Trimester of Pregnancy: A CrossSectional Study in Wuxi, China. J Trace Elements Med Biol (2017) 43:148-52. doi: 10.1016/j.jtemb.2017.01.004

16. Li S, Xin G, Wei Y, Zhu G, Yang C. The Relationship Between Iron Deficiency and Thyroid Function in Chinese Women During Early Pregnancy. J Nutr Sci Vitaminol (2017) 62:397-401. doi: 10.3177/jnsv.62.397

17. Yu X, Shan Z, Li C, Mao J, Wang W, Xie X, et al. Iron Deficiency, an Independent Risk Factor for Isolated Hypothyroxinemia in Pregnant and 
Nonpregnant Women of Childbearing Age in China. J Clin Endocrinol Metab (2015) 100:1594-601. doi: 10.1210/jc.2014-3887

18. Soliman AT, De Sanctis V, Yassin M, Wagdy M, Soliman N. Chronic Anemia and Thyroid Function. Acta BioMed (2017) 88:119-27. doi: 10.23750/ abm.v88i1.6048

19. Leslie MS, Park J, Briggs LA, El-Banna MM, Greene J. Is Anemia in Low Income Pregnant Women Related to Their Infants' Having Anemia? A Cohort Study of Pregnant Women-Infant Pairs in the United States. Matern Child Health J (2020) 24:764-76. doi: 10.1007/s10995-020-02912-8

20. Zhao J, Zhu X, Dai Q, Hong X, Zhang H, Huang K, et al. The Prevalence and Influencing Factors of Anaemia Among Pre-Pregnant Women in Mainland China: A Large Population-Based, Cross-Sectional Study. Br J Nutr (2021) 5:1-12. doi: 10.1017/S0007114521001148

21. Jiang S, Pang XH, Duan YF, Bi Y, Wang J, Yin SA, et al. The Influencing Factors of Anemia for Pregnant Women Between 2010-2012 in China. Zhonghua Yu Fang Yi Xue Za Zhi (2018) 52:21-25. Chinese. doi: 10.3760/ cma.j.issn.0253-9624.2018.01.005

22. Faul F, Erdfelder E, Lang AG, Buchner A. G*Power 3: A Flexible Statistical Power Analysis Program for the Social, Behavioral, and Biomedical Sciences. Behav Res Methods (2007) 39:175-91. doi: 10.3758/BF03193146

23. National Committee for Clinical Laboratory Standards (NCCLS). NCCLS Proposed Guideline: How to define, determine, and utilize reference intervals in the clinical laboratory Second Edition[J]. NCCLS Document C. (2000) 28-A2.

24. Zhao SY, Jing WZ, Liu J, Liu M. Prevalence of Anemia During Pregnancy in China, 2012-2016: a Meta-analysis. Zhonghua Yu Fang Yi Xue Za Zhi (2018) 52:951-7. doi: 10.3760/cma.j.issn.0253-9624.2018.09.016

25. Hu Y, Li M, Chen J, Wang R, Li W, Yang Y, et al. The Anemia and Vitamin A, Vitamin D Nutritional Status of Chinese Rural Pregnant Women in 20102012. Wei Sheng Yan Jiu (2017) 46:361-72.

26. Lin L, Wei Y, Zhu W, Wang C, Su R, Feng H, et al. Prevalence, Risk Factors and Associated Adverse Pregnancy Outcomes of Anaemia in Chinese Pregnant Women: A Multicentre Retrospective Study. BMC Pregnancy Childbirth (2018) 18:111. doi: 10.1186/s12884-018-1739-8

27. Veltri F, Decaillet S, Kleynen P, Grabczan L, Belhomme J, Rozenberg S, et al. Prevalence of Thyroid Autoimmunity and Dysfunction in Women With Iron Deficiency During Early Pregnancy: Is it Altered? Eur J Endocrinol (2016) 175:191-9. doi: 10.1530/EJE-16-0288

28. Pek LZ, Ka?Maz E, Bozaykut A, Sezer R. The Effect of Iron Deficiency Anemia on Plasma Thyroid Hormone Levels in Childhood. Turk Pediatri Arivi (2011) 46:129-32.

29. Tienboon P, Unachak K. Iron Deficiency Anaemia in Childhood and Thyroid Function. Asia Pacific J Clin Nutr (2003) 12:198-202.

30. Ravanbod M, Asadipooya K, Kalantarhormozi M, Nabipour I, Omrani GR. Treatment of Iron-Deficiency Anemia in Patients With Subclinical Hypothyroidism. Am J Med (2013) 126:420-4. doi: 10.1016/j.amjmed. 2012.12.009

31. Gauger M, Mohr W. A Rise in Haemoglobin Levels may Enhance Serum Triiodothyronine (T3). Concentrations in Prepubertal Patients With BetaThalassaemia Major. Exp Clin Endocrinol (1990) 96:169-76. doi: 10.1055/s0029-1211006

32. Hu X, Teng X, Zheng H, Shan Z, Li J, Jin T, et al. Iron Deficiency Without Anemia Causes Maternal Hypothyroxinemia in Pregnant Rats. Nutr Res (2014) 34:604-12. doi: 10.1016/j.nutres.2014.06.007

33. Khatiwada S, Gelal B, Baral N, Lamsal M. Association Between Iron Status and Thyroid Function in Nepalese Children. Thyroid Res (2016) 9:2. doi: 10.1186/ s13044-016-0031-0

34. Eftekhari MH, Simondon KB, Jalali M, Keshavarz SA, Saadat N. Effects of Administration of Iron, Iodine and Simultaneous Iron-Plus-Iodine on the
Thyroid Hormone Profile in Iron-Deficient Adolescent Iranian Girls. Eur J Clin Nutr (2006) 60:545-52. doi: 10.1038/sj.ejcn.1602349

35. Erdem G, Cengiz D, Imdat D. The Effects of Iron Deficiency Anemia on the Thyroid Functions. J Clin Exp Investigations (2010) 1:1-5. doi: 10.5799/ ahinjs.01.2010.03.0033

36. Beard JL, Borel MJ, Derr J. Impaired Thermoregulation and Thyroid Function in Iron-Deficiency Anemia. Am J Clin Nutr (1990) 52:813. doi: 10.1093/ajcn/ 52.5.813

37. Hakan C, Cemil B, Feyzi G, Talat B. Hematologic Effects of Levothyroxine in Iron-Deficient Subclinical Hypothyroid Patients: A Randomized, DoubleBlind, Controlled Study. J Clin Endocrinol Metab (2009) 94:151-6. doi: 10.1210/jc.2008-1440

38. Kennedy RL, Darne J. The Role of hCG in Regulation of the Thyroid Gland in Normal and Abnormal Pregnancy. Obstetrics Gynecol (1991) 78:298-307.

39. Weeke J, Dybkjaer L, Granlie K, Jensen SE, Magnusson B. A Longitudinal Study of Serum TSH, and Total and Free Iodothyronines During Normal Pregnancy. Acta Endocrinol (1982) 101:531. doi: 10.1530/acta.0.1010531

40. Boucai L, Surks MI. Reference Limits of Serum TSH and Free T4 are Significantly Influenced by Race and Age in an Urban Outpatient Medical Practice. Clin Endocrinol (2009) 70:788-93. doi: 10.1111/j.13652265.2008.03390.x

41. Han L, Zheng W, Zhai Y, Xie X, Zhang J, Zhang S, et al. Reference Intervals of Trimester-Specific Thyroid Stimulating Hormone and Free Thyroxine in Chinese Women Established by Experimental and Statistical Methods. J Clin Lab Anal (2018) 32:e22344. doi: 10.1002/jcla.22344

42. Gao X, Li Y, Li J, Liu A, Wei S, Teng W, et al. Gestational TSH and FT4 Reference Intervals in Chinese Women: A Systematic Review and MetaAnalysis. Front Endocrinol (2018) 9:432. doi: 10.3389/fendo.2018.00432

43. Stagnaro-Green A, Abalovich M, Alexander E, Azizi F, Mestman J, Negro R, et al. Guidelines of the American Thyroid Association for the Diagnosis and Management of Thyroid Disease During Pregnancy and Postpartum. Thyroidol (2011) 21:1081-125. doi: 10.1089/thy.2011.0087

44. Yan YQ, Dong ZL, Dong L, Wang FR, Yang XM, Jin XY, et al. Trimester- and Method-Specific Reference Intervals for Thyroid Tests in Pregnant Chinese Women: Methodology, Euthyroid Definition and Iodine Status can Influence the Setting of Reference Intervals. Clin Endocrinol (2011) 74:262-9. doi: 10.1111/j.1365-2265.2010.03910.x

45. Li C, Shan Z, Mao J, Wang W, Xie X, Zhou W, et al. Assessment of Thyroid Function During First-Trimester Pregnancy: What is the Rational Upper Limit of Serum TSH During the First Trimester in Chinese Pregnant Women? J Clin Endocrinol Metab (2014) 99:73-9. doi: 10.1210/jc.2013-1674

Conflict of Interest: The authors declare that the research was conducted in the absence of any commercial or financial relationships that could be construed as a potential conflict of interest.

Publisher's Note: All claims expressed in this article are solely those of the authors and do not necessarily represent those of their affiliated organizations, or those of the publisher, the editors and the reviewers. Any product that may be evaluated in this article, or claim that may be made by its manufacturer, is not guaranteed or endorsed by the publisher.

Copyright (c) 2021 Nie, Wang, Liu, Li and Sun. This is an open-access article distributed under the terms of the Creative Commons Attribution License (CC BY). The use, distribution or reproduction in other forums is permitted, provided the original author(s) and the copyright owner(s) are credited and that the original publication in this journal is cited, in accordance with accepted academic practice. No use, distribution or reproduction is permitted which does not comply with these terms. 